\title{
Perioperative and Long-Term Outcome for Intrahepatic Cholangiocarcinoma: Impact of Major Versus Minor Hepatectomy
}

\author{
Xu-Feng Zhang ${ }^{1,2}$ - Fabio Bagante ${ }^{3}$. Jeffery Chakedis ${ }^{2}$ - Dimitrios Moris $^{2}$. \\ Eliza W. Beal ${ }^{2} \cdot$ Matthew Weiss $^{3}$ • Irinel Popescu ${ }^{4} \cdot$ Hugo P. Marques ${ }^{5}$. \\ Luca Aldrighetti ${ }^{6}$ - Shishir K. Maithel $^{7}$ - Carlo Pulitano ${ }^{8}$ - Todd W. Bauer ${ }^{9}$. \\ Feng Shen ${ }^{10}$ • George A. Poultsides ${ }^{11}$. Oliver Soubrane ${ }^{12}$. Guillaume Martel ${ }^{13}$. \\ B. Groot Koerkamp ${ }^{14}$ • Alfredo Guglielmi ${ }^{2}$ - Endo Itaru ${ }^{15}$ - Timothy M. Pawlik ${ }^{2}$
}

Received: 28 April 2017 / Accepted: 6 July 2017 /Published online: 25 July 2017

(C) 2017 The Society for Surgery of the Alimentary Tract

\begin{abstract}
Background The objective of the current study was to investigate both short- and long-term outcomes of patients undergoing curative-intent resection for intrahepatic cholangiocarcinoma (ICC) stratified by extent of hepatic resection relative to overall final pathological margin status.

Methods One thousand twenty-three patients with ICC who underwent curative-intent resection were identified from a multiinstitutional database. Demographic, clinicopathological, and operative data, as well as overall (OS) and recurrence-free survival (RFS) were compared among patients undergoing major and minor resection before and after propensity score matching.

Results Overall, 608 (59.4\%) patients underwent major hepatectomy, while 415 (40.6\%) had a minor resection. Major hepatectomy was more frequently performed among patients who had large, multiple, and bilobar tumors. Roughly half of patients $(n=294$, $48.4 \%)$ developed a postoperative complication following major hepatectomy versus only one fourth of patients $(n=113,27.2 \%)$ after minor resection $(p<0.001)$. In the propensity model, patients who underwent major hepatectomy had an equivalent OS and RFS versus patients who had a minor hepatectomy (median OS, 38 vs. 37 months, $p=0.556$; and median RFS, 20 vs. 18 months, $p=0.635$ ). Patients undergoing major resection had comparable OS and RFS with wide surgical margin ( $\geq 10$ and $5-9 \mathrm{~mm})$, but
\end{abstract}

Electronic supplementary material The online version of this article (doi:10.1007/s11605-017-3499-6) contains supplementary material, which is available to authorized users.

Timothy M. Pawlik

tim.pawlik@osumc.edu

1 Department of Hepatobiliary Surgery and Institute of Advanced Surgical Technology and Engineering, The First Affiliated Hospital of Xi'an Jiaotong University, Xi'an, China

2 Department of Surgery, The Ohio State University Wexner Medical Center, 395 W. 12th Ave., Suite, Columbus, OH 670, USA

3 Department of Surgery, Johns Hopkins Hospital, Baltimore, MD, USA

4 Department of Surgery, Fundeni Clinical Institute, Bucharest, Romania

5 Department of Surgery, Curry Cabral Hospital, Lisbon, Portugal

6 Department of Surgery, Ospedale San Raffaele, Milan, Italy

7 Department of Surgery, Emory University, Atlanta, GA, USA
8 Department of Surgery, Royal Prince Alfred Hospital, University of Sydney, Sydney, Australia

9 Department of Surgery, University of Virginia, Charlottesville, VA, USA

10 Department of Surgery, Eastern Hepatobiliary Surgery Hospital, Shanghai, China

11 Department of Surgery, Stanford University, Stanford, CA, USA

12 Department of Hepatobiliopancreatic Surgery and Liver Transplantation, AP-HP, Beaujon Hospital, Clichy, France

13 Division of General Surgery, Department of Surgery, University of Ottawa, Ottawa, ON, Canada

14 Department of Surgery, Erasmus University Medical Centre, Rotterdam, Netherlands

15 Gastroenterological Surgery Division, Yokohama City University School of Medicine, Yokohama, Japan 
improved RFS when surgical margin was narrow $(1-4 \mathrm{~mm}$ ) versus minor resection in the propensity model. In the Cox regression model, tumor characteristics and surgical margin were independently associated with long-term outcome.

Conclusions Major hepatectomy for ICC was not associated with an overall survival benefit, yet was associated with increased perioperative morbidity. Margin width, rather than the extent of resection, affected long-term outcomes. Radical parenchymalsparing resection should be advocated if a margin clearance of $\geq 5 \mathrm{~mm}$ can be achieved.

Keywords Intrahepatic cholangiocarcinoma $\cdot$ Major · Minor · Hepatectomy $\cdot$ Outcomes

\section{Introduction}

Intrahepatic cholangiocarcinoma (ICC) is the second most common type of primary liver cancer after hepatocellular carcinoma (HCC), accounting for 5-30\% of all primary hepatic malignancies. ${ }^{1-3}$ ICC originates from malignant transformation of intrahepatic biliary epithelial cells. While rare, the incidence of ICC has increased from 0.44 to 1.18 cases per 100,000 between 1973 and 2012 in the USA with the incidence continuing to increase worldwide. ${ }^{4}$ The rise in the reported number of ICC cases is undoubtedly multifactorial and may be due to better imaging, more disease-specific histopathologic techniques, as well as an increase in the prevalence of certain risk factors. ${ }^{4,5}$ While rare, ICC is an aggressive disease with outcomes that are typically worse than HCC.,

For patients with ICC, hepatic resection remains the best therapeutic approach; however, less than $40 \%$ of ICC patients are potential candidates for resection due to advanced disease at presentation. ${ }^{5,7}$ Among patients who are candidates for surgery, 5-year survival following curative-intent resection ranges from only 30 to $50 \%$. $^{2}$ Both biological and technical factors are associated with survival after resection of ICC. In particular, lymph node metastasis, tumor size, number, and grade are all associated with long-term outcomes. ${ }^{8-11}$ In addition, our group had previously reported that R1 margin status was associated with inferior long-term outcomes. ${ }^{8}$ In fact, we reported that there was an incremental worsening of recurrence-free and overall survival as margin width decreased. Obtaining a wider surgical margin often necessitates, however, a more extensive hepatic resection. While major hepatectomy, defined as resection of three or more liver segments, ${ }^{12}$ may facilitate a wider surgical margin and potential removal of tumor-bearing portal tributaries, perioperative morbidity and mortality may be higher compared with minor resection. ${ }^{13-15}$ While previous studies have advocated for parenchymal sparing, minor resections for diseases such as colorectal liver metastasis, other reports have noted an improvement in outcomes following large, anatomic resections of other diseases such as HCC. ${ }^{13,16-18}$ The impact of resection extent, specifically the type of liver resection (i.e., minor vs. major), on outcomes following resection of ICC has not been examined. Given the lack of data on long-term outcomes following minor versus major hepatectomy for ICC, the objective of the current study was to investigate both short- and long-term outcomes stratified by extent of hepatic resection relative to overall final pathological margin status. In addition, we sought to define the relative survival benefit of achieving additional margin width compared with any potential detrimental complication-related effects on long-term prognosis.

\section{Materials and Methods}

\section{Study Population}

A multi-institutional database consisting of 1142 patients undergoing curative-intent hepatic resection for ICC from 1990 to 2016 at 14 major hepatobiliary centers in America, Europe, Australia, and Asia were identified. The 14 medical centers included The Ohio State University, Columbus, OH; Stanford University, Stanford, CA; University of Virginia, Charlottesville, VA; Emory University, Atlanta, GA; Fundeni Clinical Institute of Digestive Disease, Bucharest, Romania; Johns Hopkins Hospital, Baltimore, MD; Curry Cabral Hospital, Lisbon, Portugal; Ospedale San Raffaele, Milan, Italy; Royal Prince Alfred Hospital, University of Sydney, Sydney, Australia; Eastern Hepatobiliary Surgery Hospital, Shanghai, China; Beaujon Hospital, Clichy, France; University of Ottawa, Ottawa, Ontario, Canada; Erasmus University Medical Centre, Rotterdam, Netherlands; and Yokohama City University School of Medicine, Yokohama, Japan. All patients were diagnosed with ICC as confirmed by histological examination. Patients with extrahepatic metastasis were excluded. Patients undergoing an R2 resection $(n=13)$, ablation only $(n=2)$, or other intra-arterial therapies $(n=15)$ were also excluded. Moreover, 89 patients with insufficient details on resection type and/or survival time were excluded. After excluding patients based on these criteria, 1023 patients remained in the analytic cohort. The Institutional Review Board of each participating institution approved the study.

\section{Data Collection}

Demographic and clinicopathologic data were collected for each patient including age, gender, ethnicity, American Society of Anesthesiologists (ASA) score, body mass index (BMI), as well as the presence of clinical jaundice or liver cirrhosis. Operative details included type and extent of 
Table 1 Demographic and clinicopathological characteristics of patients undergoing minor and major hepatectomy for intrahepatic cholangiocarcinoma

\begin{tabular}{|c|c|c|c|}
\hline & $\begin{array}{l}\text { Minor } \\
\text { hepatectomy } \\
(n=415)\end{array}$ & $\begin{array}{l}\text { Major } \\
\text { hepatectomy } \\
(n=608)\end{array}$ & $\begin{array}{l}p \\
\text { value }\end{array}$ \\
\hline Age (years) & $57(48-65)$ & $61(53-69)$ & $<0.001$ \\
\hline Sex & & & $<0.001$ \\
\hline Male & $259(62.4 \%)$ & $310(51.0 \%)$ & \\
\hline Female & $156(37.6 \%)$ & $297(48.8 \%)$ & \\
\hline Race & & & $<0.001$ \\
\hline White & $136(32.8 \%)$ & $403(66.3 \%)$ & \\
\hline Black & $7(1.7 \%)$ & $28(4.6 \%)$ & \\
\hline Asian & $265(63.9 \%)$ & $134(22.0 \%)$ & \\
\hline Other & $2(0.5 \%)$ & $20(3.3 \%)$ & \\
\hline Missing & $5(1.2 \%)$ & $22(3.6 \%)$ & \\
\hline Body mass index & $24.8(22.1-27.8)$ & $25.3(22.5-28.0)$ & 0.243 \\
\hline $\begin{array}{l}\text { Clinical jaundice } \\
\text { present }\end{array}$ & $13(3.1 \%)$ & $89(14.6 \%)$ & $<0.001$ \\
\hline Liver cirrhosis & $88(21.2 \%)$ & $20(3.3 \%)$ & $<0.001$ \\
\hline $\begin{array}{l}\text { Carbohydrate antigen } \\
19-9(\mathrm{U} / \mathrm{mL})\end{array}$ & $27.0(11.2-86.4)$ & $88.0(24.8-341.3)$ & $<0.001$ \\
\hline $\begin{array}{l}\text { Carcinoembryonic } \\
\text { antigen }(\mathrm{ng} / \mathrm{mL})\end{array}$ & $2.3(1.4-3.5)$ & $2.4(1.3-4.6)$ & 0.257 \\
\hline Tumor size $(\mathrm{cm})$ & $5.0(3.6-7.3)$ & $7.0(4.7-9.5)$ & $<0.001$ \\
\hline Multiple lesions $(\geq 2)$ & $51(12.3 \%)$ & $125(20.6 \%)$ & 0.001 \\
\hline Bilobar tumor & $40(9.6 \%)$ & $150(24.7 \%)$ & $<0.001$ \\
\hline \multicolumn{4}{|l|}{ Vascular invasion } \\
\hline Macro & $24(5.8 \%)$ & $87(14.3 \%)$ & $<0.001$ \\
\hline Micro & $57(13.7 \%)$ & $224(36.8 \%)$ & $<0.001$ \\
\hline Perineural invasion & $25(6.0 \%)$ & $133(21.9 \%)$ & $<0.001$ \\
\hline $\begin{array}{l}\text { Direct invasion of } \\
\text { adjacent organs }\end{array}$ & $17(4.1 \%)$ & $58(9.5 \%)$ & 0.001 \\
\hline Biliary invasion & $12(2.9 \%)$ & $125(20.6 \%)$ & $<0.001$ \\
\hline Satellite lesions & $75(18.1 \%)$ & $148(24.3 \%)$ & 0.021 \\
\hline AJCC T stage & & & $<0.001$ \\
\hline $\mathrm{T} 1-\mathrm{T} 2$ & $372(89.6 \%)$ & $404(66.4 \%)$ & \\
\hline $\mathrm{T} 3-\mathrm{T} 4$ & $26(6.3 \%)$ & $147(24.2 \%)$ & \\
\hline Missing & $17(4.1 \%)$ & $57(9.4 \%)$ & \\
\hline AJCC N stage & & & $<0.001$ \\
\hline N0 & $268(64.6 \%)$ & $294(48.4 \%)$ & \\
\hline N1-N2 & $40(9.6 \%)$ & $138(22.7 \%)$ & \\
\hline $\mathrm{Nx}$ & $107(25.8 \%)$ & $176(28.9 \%)$ & \\
\hline Histological grade & & & $<0.001$ \\
\hline Well to moderate & $365(88.0 \%)$ & $446(73.4 \%)$ & \\
\hline Poor to undifferentiated & $34(8.2 \%)$ & $138(22.7 \%)$ & \\
\hline Missing & $16(3.9 \%)$ & $24(3.9 \%)$ & \\
\hline Morphological type & & & $<0.001$ \\
\hline Mass forming & $386(93.0 \%)$ & $431(70.9 \%)$ & \\
\hline Papillary & $9(2.2 \%)$ & $19(3.1 \%)$ & \\
\hline Periductal infiltrating & $3(0.7 \%)$ & $46(7.6 \%)$ & \\
\hline $\begin{array}{l}\text { Mass-forming }+ \\
\text { periductal infiltrating }\end{array}$ & $6(1.4 \%)$ & $66(10.9 \%)$ & \\
\hline Missing & $11(2.7 \%)$ & $46(7.6 \%)$ & \\
\hline \multicolumn{4}{|l|}{ Resection procedure } \\
\hline Wedge resection & $161(38.8 \%)$ & - & \\
\hline Monosegmentectomy & $79(19.0 \%)$ & - & \\
\hline Bisegmentectomy & $175(42.2 \%)$ & - & \\
\hline Trisegmentectomy & - & $18(3.0 \%)$ & \\
\hline Right hepatectomy & - & $161(26.5 \%)$ & \\
\hline Left hepatectomy & - & $202(33.2 \%)$ & \\
\hline $\begin{array}{l}\text { Extended right } \\
\text { hepatectomy }\end{array}$ & - & $128(21.1 \%)$ & \\
\hline $\begin{array}{l}\text { Extended left } \\
\text { hepatectomy }\end{array}$ & - & $99(16.3 \%)$ & \\
\hline R0 resection & $389(93.7 \%)$ & $505(83.1 \%)$ & $<0.001$ \\
\hline
\end{tabular}

Table 1 (continued)

\begin{tabular}{llll}
\hline & $\begin{array}{l}\text { Minor } \\
\text { hepatectomy } \\
(n=415)\end{array}$ & $\begin{array}{l}\text { Major } \\
\text { hepatectomy } \\
(n=608)\end{array}$ & $\begin{array}{l}p \\
\text { value }\end{array}$ \\
\hline $\begin{array}{llll}\text { Margin distance (mm) } \\
\quad 1\end{array}$ & $26(6.3 \%)$ & $103(16.9 \%)$ & $<0.001$ \\
$1-4$ & $157(37.8 \%)$ & $192(31.6 \%)$ & \\
$5-9$ & $105(25.3 \%)$ & $110(18.1 \%)$ & \\
$\geq 10$ & $116(28.0 \%)$ & $146(24.0 \%)$ & \\
$\quad$ Missing & $11(2.7 \%)$ & $57(9.4 \%)$ & \\
Major vascular resection & $19(4.6 \%)$ & $105(17.3 \%)$ & $<0.001$ \\
$\begin{array}{l}\text { Bile duct resection } \\
\text { Lymphadenectomy }\end{array}$ & $12(2.9 \%)$ & $165(27.1 \%)$ & $<0.001$ \\
Intraoperative blood loss & $108(26.0 \%)$ & $374(61.5 \%)$ & $<0.001$ \\
Intraoperative blood & $250(150-445)$ & $600(300-1025)$ & $<0.001$ \\
$\quad$ transfusion & $55(13.3 \%)$ & $210(34.5 \%)$ & $<0.001$ \\
Postoperative blood & $12(2.9 \%)$ & $77(12.7 \%)$ & $<0.001$ \\
$\quad$ transfusion & $120(90-180)$ & $297(200-420)$ & $<0.001$ \\
Operation time (min) & & & \\
\hline
\end{tabular}

hepatectomy, type and extent of vascular resection, receipt of lymphadenectomy, margin status, operative time, estimated blood loss, and intraoperative blood transfusion. Tumor size, number, morphology, vascular/perineural/biliary/adjacent organ invasion, lymph node metastasis, histological grade, and resection margin were ascertained based on the final pathology report. Types of hepatic resection were defined according to the consensus classification. ${ }^{12}$ Major hepatectomy was defined as the resection of three or more segments (right hepatectomy, left hepatectomy, extended right hepatectomy, extended left hepatectomy, and any trisegmentectomy), whereas minor resection included resection of two or fewer segments and non-anatomic wedge resection according to the classification of Couinaud. ${ }^{19}$ Data on tumor stage were collected according to the American Joint Committee on Cancer (AJCC) 7th edition staging system. ${ }^{20}$

Primary outcomes were overall survival (OS) and recurrence-free survival (RFS), both calculated from the date of surgery. Recurrence was defined as identification of suspicious imaging findings or biopsy-proven tumor. Recurrent sites were divided as intrahepatic and/or extrahepatic. Secondary outcomes were postoperative morbidity and mortality at 30 and 90 days following surgery. Postoperative complications were graded according to the Clavien-Dindo classification (CDC) of surgical complication. ${ }^{21}$

\section{Statistical Analysis}

Numerical variables were expressed as medians with interquartile ranges (IQR) and compared with student $t$ test or Mann-Whitney $U$ test between the two groups. Nominal variables were expressed as number and percentages and compared with Chi-squared test or Fisher's exact test. KaplanMeier survival analyses were used to compare survival using the log-rank test between any two groups. Potential risk factors associated with OS and RFS were identified using 
univariate and multivariable Cox hazard regression models after exclusion of patients who died within 90 days postoperatively $(n=60)$, and expressed as hazard ratio (HR) and $95 \%$ confidence interval (CI). Factors with $p$ value $<0.1$ by univariate analysis were included in multivariable analysis.

Since patients who underwent major and minor hepatectomy groups were not randomly distributed, propensity score matching (PSM) was used to mitigate selection bias. Specifically, variables potentially affecting long-term outcomes were utilized in the propensity score based on identification in logistic regression analysis. Propensity score analysis with 1:1 matching was performed without replacement using a caliper with a width 0.1 of the standard deviation to generate matched pairs of the patients. In all analyses, two-tailed $p$ value $<0.05$ was considered statistically significant. Statistical analysis was carried out using SPSS 22.0 (Chicago, IL, USA).

\section{Results}

\section{Baseline Demographic and Clinicopathological Characteristics}

The median age of the 1023 patients with ICC was 59 years (IQR, 51-68 years); the majority were male $(n=569,55.6 \%)$ and Caucasian $(n=539,52.7 \%)$. Overall, $608(59.4 \%)$ patients underwent major hepatectomy, while 415 (40.6\%) had a minor resection. Patients undergoing major hepatectomy were older, more likely to be female, and more often presented with clinical jaundice (Table 1). Major hepatectomy was more frequently performed among patients who had large, multiple, and bilobar tumors. Patients undergoing major hepatectomy were also more likely to have advanced disease characterized by vascular, biliary, perineural, and adjacent tissue invasion, as well as poor tumor differentiation (Table 1). Interestingly, patients who underwent a major hepatectomy were more likely to have a microscopically positive (R1) margin than patients who underwent minor resection $(p<0.001$, Table 1$)$.

\section{Association of Resection Extent with Short- and Long-Term Outcomes}

Not surprisingly, major hepatectomy was associated with longer operative times, a larger volume of intraoperative blood loss, and more frequent intra- and postoperative transfusion of packed red blood cells (Table 1). Consequently, almost one half of patients ( $n=294,48.4 \%$ ) developed a postoperative complications following major hepatectomy versus only one fourth of patients $(n=113,27.2 \%)$ after minor resection $(p<0.001)$. Moreover, major complications (CDC III-IV) and postoperative death $(\mathrm{CDC} \mathrm{V})$ were more common after a major versus minor hepatic resection (both $p<0.01$ ), while minor complications (CDC I-II) were comparable ( $p=0.303$, Table 2). Major hepatectomy was associated with more frequent surgical technique-related (anastomotic leakage, intra-abdominal abscess, liver failure, cholangitis, and bowel perforation/ileus), medical (respiratory insufficiency, pulmonary embolism, renal failure, and cardiac events), and infectious (systemic, urinary

Table 2 Short- and long-term outcome of patients undergoing minor and major hepatectomy for intrahepatic cholangiocarcinoma

\begin{tabular}{|c|c|c|c|}
\hline & $\begin{array}{l}\text { Minor } \\
\text { hepatectomy } \\
(n=415)\end{array}$ & $\begin{array}{l}\text { Major } \\
\text { hepatectomy } \\
(n=608)\end{array}$ & $\begin{array}{l}p \\
\text { value }\end{array}$ \\
\hline $\begin{array}{l}\text { Postoperative } \\
\text { complications }\end{array}$ & $113(27.2 \%)$ & $294(48.4 \%)$ & $<0.001$ \\
\hline $\begin{array}{l}\text { Clavien-Dindo } \\
\text { classification }\end{array}$ & & & $<0.001$ \\
\hline I & $51(12.3 \%)$ & $54(8.9 \%)$ & \\
\hline II & $32(7.7 \%)$ & $84(13.8 \%)$ & \\
\hline III & $24(5.8 \%)$ & $105(17.3 \%)$ & \\
\hline IV & 0 & $12(2.0 \%)$ & \\
\hline $\mathrm{V}$ & $6(1.4 \%)$ & $39(6.4 \%)$ & \\
\hline \multicolumn{4}{|l|}{ Gastrointestinal } \\
\hline $\begin{array}{l}\text { Anastomotic/bile } \\
\text { leakage }\end{array}$ & $13(3.1 \%)$ & $65(10.7 \%)$ & $<0.001$ \\
\hline Intra-abdominal abscess & $2(0.5 \%)$ & $27(4.4 \%)$ & $<0.001$ \\
\hline Liver abscess & $2(0.5 \%)$ & $6(1.0 \%)$ & 0.484 \\
\hline Cholangitis & 0 & $7(1.2 \%)$ & 0.046 \\
\hline Liver failure & $3(0.7 \%)$ & $24(3.9 \%)$ & 0.001 \\
\hline Portal vein thrombosis & 0 & $4(0.7 \%)$ & 0.151 \\
\hline $\begin{array}{l}\text { Intra-abdominal/GI } \\
\text { bleeding }\end{array}$ & $7(1.7 \%)$ & $17(2.8 \%)$ & 0.297 \\
\hline Bowel perforation/ileus & $1(0.2 \%)$ & $9(1.5 \%)$ & 0.055 \\
\hline \multicolumn{4}{|l|}{ Pulmonary } \\
\hline Pleural effusion & $38(9.2 \%)$ & $47(7.7 \%)$ & 0.422 \\
\hline Pneumothorax & 0 & $3(0.5 \%)$ & 0.276 \\
\hline $\begin{array}{l}\text { Respiratory } \\
\text { insufficiency }\end{array}$ & $3(0.7 \%)$ & $20(3.3 \%)$ & 0.008 \\
\hline Pneumonia & $7(1.7 \%)$ & $12(2.0 \%)$ & 0.817 \\
\hline Pulmonary embolism & $1(0.2 \%)$ & $9(1.5 \%)$ & 0.055 \\
\hline Cardiac events & $4(1.0 \%)$ & $22(3.6 \%)$ & 0.008 \\
\hline \multicolumn{4}{|l|}{ Urinary } \\
\hline Urinary tract infection & 0 & $13(2.1 \%)$ & 0.001 \\
\hline Renal failure & $1(0.2 \%)$ & $11(1.8 \%)$ & 0.034 \\
\hline Systemic sepsis & $3(0.7 \%)$ & $27(4.4 \%)$ & $<0.001$ \\
\hline $\begin{array}{l}\text { Wound } \\
\text { infection/dehiscence }\end{array}$ & $3(0.7 \%)$ & $27(4.4 \%)$ & $<0.001$ \\
\hline Other complications & $33(8.0 \%)$ & $49(8.1 \%)$ & 1.000 \\
\hline $\begin{array}{l}\text { Readmission within } \\
30 \text { days }\end{array}$ & $5(1.2 \%)$ & $43(7.1 \%)$ & $<0.001$ \\
\hline 30-day mortality & $6(1.4 \%)$ & $43(7.1 \%)$ & $<0.001$ \\
\hline 90-day mortality & $13(3.1 \%)$ & $47(7.7 \%)$ & 0.002 \\
\hline Tumor recurrence & & & 0.103 \\
\hline Intrahepatic & $195(47.0 \%)$ & $234(38.8 \%)$ & \\
\hline Extrahepatic & $21(5.1 \%)$ & $43(7.1 \%)$ & \\
\hline Intra- and extrahepatic & $46(11.1 \%)$ & $72(11.8 \%)$ & \\
\hline
\end{tabular}


tract, and wound infection) complications (Table 2). In turn, readmission and perioperative mortality were higher among patients after major versus minor hepatectomy (all $p<0.01$ ).

Among all patients, median, 1-, 3-, and 5-year OS was 37 months, $78 \%, 51 \%$, and 39\%, respectively. During followup, $611(60 \%)$ patients experienced a tumor recurrence; median, 1-, 3-, and 5-year RFS was 17 months, 57\%, 36\%, and 31\%, respectively. The recurrence site was intrahepatic tumor only in most patients ( $n=429 / 611 ; 70 \%)$ (Table 2). Interestingly, patients undergoing major liver resection had a worse OS, yet a similar RFS compared with patients who underwent a minor resection (median OS, 34 vs. 49 months, $p=0.004$; median RFS, 16 vs. 19 months, $p=0.151$; Fig. 1a, b). A Cox regression model was performed to identify risk factors associated with overall and recurrence-free survival of 963 patients after exclusion of patients who died within 90 days following surgery $(n=60)$. On multivariable analysis, tumor characteristics, surgical margin width, and intraoperative blood transfusion were associated with OS and RFS, yet not resection extent (major vs. minor) (Tables 3 and 4).

a

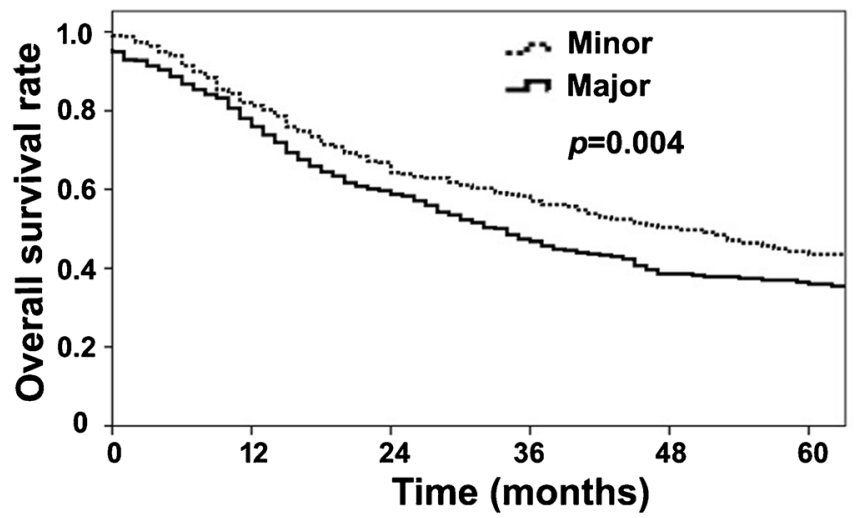

Given the baseline differences in the minor versus major hepatectomy cohorts, PSM was then utilized to generate 290 pairs of well-matched patients with similar tumor size, number, distribution, vascular invasion, surgical margin width, incidence and severity of postoperative complications, and in hospital mortality (Supplementary Table 1). In the propensity model, patients who underwent major hepatectomy had equivalent OS and RFS as patients who had a minor hepatectomy (median OS, 38 vs. 37 months, $p=0.556$; and median RFS, 20 vs. 18 months, $p=0.635$, Fig. 1c, d).

\section{Outcomes of Patients with Different Width of Surgical Margin}

Given that surgical margin may be associated with the extent of resection, patients who underwent $\mathrm{R} 0$ resection were stratified into three groups with different margin width: $\geq 10,5-9$, and 1-4 $\mathrm{mm}$. The impact of major versus minor resection on OS and RFS using PSM was then assessed (Supplementary

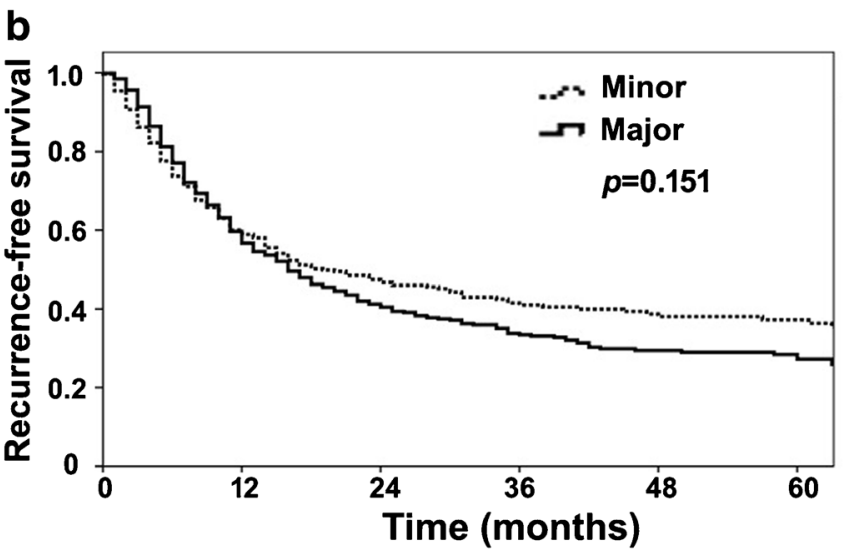

After propensity socre matching
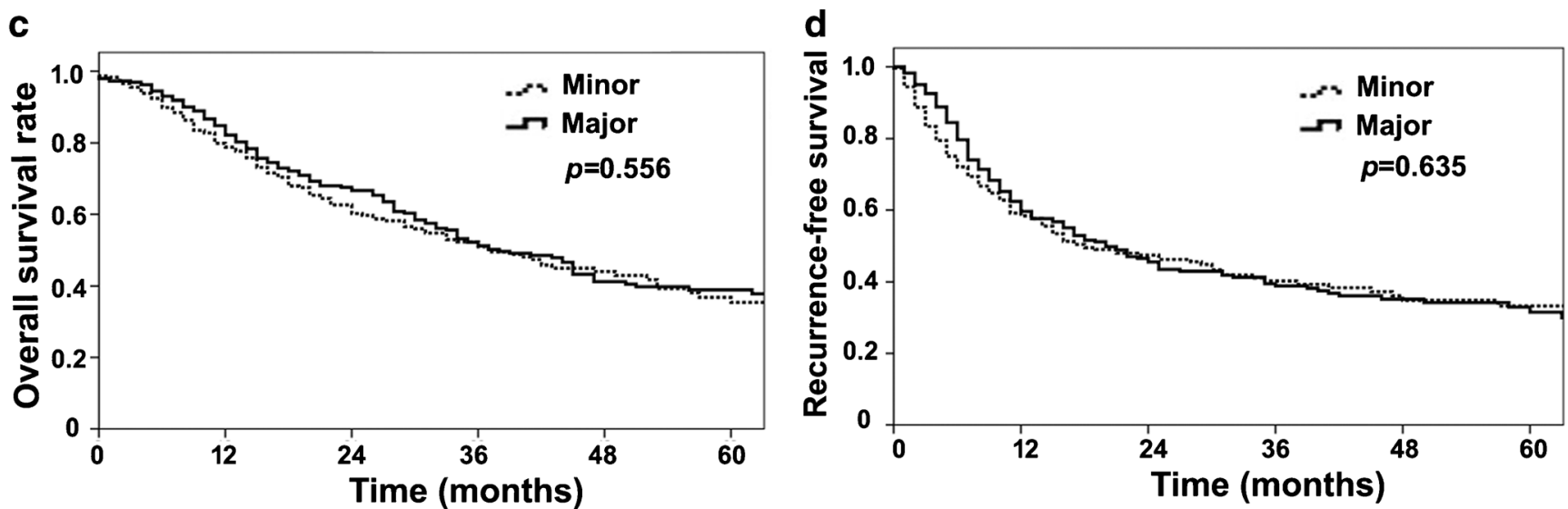

Fig. 1 Overall survival (a, c) and recurrence-free survival (b, d) before and after propensity score matching of patients undergoing major and minor hepatectomy for intrahepatic cholangiocarcinoma 
Table 3 Univariate and multivariable analysis of risk factors associated with overall survival of ICC patients $(n=963)$ after exclusion of 90-day mortality in Cox hazard regression model

\begin{tabular}{|c|c|c|c|c|}
\hline \multirow[t]{2}{*}{ Variables } & \multicolumn{2}{|c|}{ Univariate analysis } & \multicolumn{2}{|c|}{ Multivariate analysis } \\
\hline & HR $(95 \% \mathrm{CI})$ & $p$ value & HR $(95 \% \mathrm{CI})$ & $p$ value \\
\hline Sex (male/female) & $0.9(0.7-1.1)$ & 0.209 & & \\
\hline Age $(>55 / \leq 55$ years $)$ & $0.9(0.8-1.1)$ & 0.518 & & \\
\hline \multicolumn{5}{|l|}{ Tumor size $(\mathrm{cm})$} \\
\hline$\leq 5$ & Reference & & Reference & \\
\hline $5-10$ & $1.8(1.4-2.2)$ & $<0.001$ & $1.0(0.6-1.4)$ & 0.991 \\
\hline$\geq 10$ & $1.8(1.4-2.4)$ & $<0.001$ & $1.0(0.6-1.8)$ & 0.845 \\
\hline Multiple tumors & $2.0(1.6-2.5)$ & $<0.001$ & $1.5(0.9-2.2)$ & 0.088 \\
\hline Bilobar & $1.4(1.1-1.7)$ & 0.006 & $1.0(0.7-1.5)$ & 0.985 \\
\hline Vascular invasion & $1.6(1.2-2.2)$ & $<0.001$ & $1.3(0.8-2.2)$ & 0.354 \\
\hline Perineural invasion & $1.4(1.1-1.8)$ & 0.013 & $1.3(0.9-1.9)$ & 0.401 \\
\hline Biliary invasion & $1.5(1.1-1.9)$ & 0.004 & $0.9(0.6-1.3)$ & 0.325 \\
\hline Direct invasion of adjacent organs & $2.4(1.7-3.2)$ & $<0.001$ & $1.1(0.7-2.6)$ & 0.619 \\
\hline Satellite lesions & $2.1(1.7-2.6)$ & $<0.001$ & $1.0(0.6-1.5)$ & 0.924 \\
\hline Lymph nodes metastasis & $3.2(2.5-4.2)$ & $<0.001$ & $2.8(2.0-4.0)$ & $<0.001$ \\
\hline Poor to undifferentiated & $1.6(1.3-2.1)$ & $<0.001$ & $2.0(1.4-2.9)$ & $<0.001$ \\
\hline Major hepatectomy & $1.2(1.0-1.5)$ & 0.058 & $1.0(0.6-1.7)$ & 0.908 \\
\hline \multicolumn{5}{|l|}{ Margin width (mm) } \\
\hline$\geq 10$ & Reference & & Reference & \\
\hline $5-9$ & $1.0(0.8-1.3)$ & 0.966 & $1.2(0.7-2.0)$ & 0.557 \\
\hline $1-4$ & $1.3(1.1-1.7)$ & 0.016 & $1.9(1.1-3.3)$ & 0.016 \\
\hline$<1$ & $1.7(1.3-2.4)$ & 0.001 & $1.9(1.2-3.2)$ & 0.007 \\
\hline Intraoperative blood transfusion & $2.1(1.8-2.6)$ & $<0.001$ & $1.5(1.1-2.2)$ & 0.024 \\
\hline Postoperative blood transfusion & $1.5(1.1-2.0)$ & 0.015 & $1.3(0.8-2.0)$ & 0.321 \\
\hline Postoperative morbidity & & 0.344 & & \\
\hline No complication & Reference & & & \\
\hline Clavien-Dindo grade I-II & $1.0(0.8-1.2)$ & & & \\
\hline Clavien-Dindo grade III- IV & $1.2(0.9-1.6)$ & & & \\
\hline
\end{tabular}

$P$ values from the multivariable models which are significant are in italics
Table 2). Patients with a wide surgical margin ( $\geq 10$ or $5-9 \mathrm{~mm})$ demonstrated no difference in OS and RFS when stratified by major versus minor resection before and after PSM (Supplementary Fig. 1A, B, Fig. 2a, b). Interestingly, patients who underwent a major resection with a narrow surgical margin (1-4 mm) had a worse OS (Supplementary Fig. 1C). However, after PSM, patients who underwent a major hepatectomy with a narrow surgical margin were noted to have a comparable OS versus patients undergoing a minor hepatectomy with close margins. In contrast, patients who underwent a major hepatectomy with close surgical margins had an improved RFS versus minor resection after matching for tumor characteristics, postoperative complications, and mortality on PSM (Fig. 2c).

\section{Discussion}

Surgical resection is the best curative treatment option for ICC and may provide patients with a chance for long-term survival. ${ }^{8,22,23}$ At the time of surgery, the extent of liver resection for a liver tumor is typically determined by tumor size, number, and location, as well as underlying quality of the non-tumorous liver. ${ }^{24,25}$ Whether major hepatectomy is necessary and superior to minor resection in improving the long-term survival of patients with HCC and CRLM has been a long-standing topic of interest. ${ }^{13-16,26}$ Major hepatectomy may be associated with wider surgical margins and theoretical removal of tumor-bearing portal tributaries, yet it sacrifices a large volume of functional liver parenchyma with higher postoperative morbidity and mortality than minor hepatectomy. ${ }^{13-15}$ The extent of hepatic resection and its impact on outcomes among patients with ICC has not been well examined. The current study is important because it specifically investigated the impact of extent of resection on both short- and long-term outcomes, as well as assessed the influence of resection extent relative to margin width using both unadjusted and PSM analyses. Of note, extent of hepatic resection did not impact OS among patients with a wider 
Table 4 Univariate and multivariable analysis of risk factors associated with recurrence-free survival of ICC patients $(n=963)$ after exclusion of 90-day mortality in Cox hazard regression model

\begin{tabular}{|c|c|c|c|c|}
\hline \multirow[t]{2}{*}{ Variables } & \multicolumn{2}{|c|}{ Univariate analysis } & \multicolumn{2}{|c|}{ Multivariate analysis } \\
\hline & HR $(95 \% \mathrm{CI})$ & $p$ value & HR $(95 \% \mathrm{CI})$ & $p$ value \\
\hline Sex (male/female) & $1.0(0.8-1.2)$ & 0.986 & & \\
\hline Age ( $>55 / \leq 55$ years) & $0.8(0.7-0.9)$ & 0.005 & $0.7(0.5-0.9)$ & 0.012 \\
\hline \multicolumn{5}{|l|}{ Tumor size $(\mathrm{cm})$} \\
\hline$\leq 5$ & Reference & & Reference & \\
\hline $5-10$ & $1.9(1.5-2.3)$ & $<0.001$ & $1.3(0.8-2.2)$ & 0.322 \\
\hline$\geq 10$ & $2.0(1.5-2.6)$ & $<0.001$ & $1.5(1.0-2.2)$ & 0.030 \\
\hline Multiple tumors & $1.8(1.5-2.2)$ & $<0.001$ & $1.7(1.2-2.4)$ & 0.007 \\
\hline Bilobar & $1.2(0.9-1.4)$ & 0.152 & & \\
\hline Vascular invasion & $1.3(1.0-1.7)$ & 0.023 & $1.6(1.0-2.5)$ & 0.062 \\
\hline Perineural invasion & $1.4(1.1-1.7)$ & 0.006 & $1.9(1.3-2.6)$ & $<0.001$ \\
\hline Biliary invasion & $1.3(1.0-1.6)$ & 0.070 & $1.0(0.7-1.4)$ & 0.910 \\
\hline Direct invasion of adjacent organs & $1.6(1.2-2.3)$ & 0.002 & $1.4(0.8-2.4)$ & 0.191 \\
\hline Satellite lesions & $2.0(1.6-2.3)$ & $<0.001$ & $1.0(0.7-1.4)$ & 0.869 \\
\hline Lymph nodes metastasis & $2.0(1.6-2.6)$ & $<0.001$ & $1.6(1.2-2.2)$ & 0.003 \\
\hline Poor to undifferentiated & $1.4(1.2-1.7)$ & 0.001 & $1.4(1.0-1.9)$ & 0.062 \\
\hline Major hepatectomy & $1.1(1.0-1.4)$ & 0.121 & & \\
\hline \multicolumn{5}{|l|}{ Margin width (mm) } \\
\hline$\geq 10$ & Reference & & Reference & \\
\hline $5-9$ & $1.2(0.9-1.5)$ & 0.162 & $1.4(0.9-2.2)$ & 0.107 \\
\hline $1-4$ & $1.4(1.1-1.7)$ & 0.005 & $1.5(1.0-2.2)$ & 0.069 \\
\hline$<1$ & $1.7(1.3-2.2)$ & $<0.001$ & $1.6(1.0-2.5)$ & 0.061 \\
\hline Intraoperative blood transfusion & $1.6(1.3-1.9)$ & $<0.001$ & $1.4(1.0-2.0)$ & 0.030 \\
\hline Postoperative blood transfusion & $1.1(0.8-1.5)$ & 0.435 & & \\
\hline Postoperative morbidity & & 0.799 & & \\
\hline No complication & Reference & & & \\
\hline $\mathrm{I}-\mathrm{II}$ & $0.9(0.7-1.1)$ & & & \\
\hline III- IV & $1.1(0.9-1.4)$ & & & \\
\hline
\end{tabular}

$P$ values from the multivariable models which are significant are in italics surgical margin ( $\geq 5 \mathrm{~mm}$ ). Therefore, the findings of the present study suggest that a radical resection with parenchymal preservation should be recommended whenever possible, as long as an adequate margin width ( $\geq 5 \mathrm{~mm}$ ) can be achieved.

A major hepatectomy has been advocated for HCC tumors $>5 \mathrm{~cm}$, as a more extensive resection allows for removal of a wider surgical margin, as well as any microscopic disease along the portal tributaries. ${ }^{27}$ Because a large portion of the resected liver is typically occupied by the large tumor mass, loss of functional liver parenchyma is typically minimal compared with major hepatectomy for small HCC. ${ }^{26}$ For small HCC $(<5 \mathrm{~cm})$, the role of major hepatectomy remains controversial. ${ }^{26,28-30}$ In fact, several studies have noted that preservation of liver parenchyma should take priority, and minor hepatectomy can provide equal OS and RFS compared with major hepatectomy for small HCC. ${ }^{16,31}$ For patients with CRLM, several studies have noted that the extent of hepatectomy is not associated with outcomes, with most studies favoring parenchymal-sparing operations for CRLM. ${ }^{13,18,32,33}$
In the current study specifically investigating ICC patients, major hepatectomy was performed in the majority (59\%), including hemihepatectomy (36\%) or extended hemihepatectomy (22\%). Of note, the utilization of major hepatectomy for ICC was higher than that reported in many studies on HCC (5$39 \%)^{26,34}$ The reason for this is likely multifactorial and may be related to the more aggressive nature of ICC and the infrequency of liver cirrhosis (10\%), which allowed for more major resections without the fear of liver insufficiency.

Notably, almost one half of the patients (48.4\%) undergoing major resection experienced a postoperative complication versus only $27 \%$ of patients who underwent a minor resection. Of note, one fourth of patients had a severe complication (CDC $\geq$ III) after a major hepatectomy.

The occurrence of a postoperative complication has previously been reported to be an independent predictor of worse long-term outcomes. ${ }^{35}$ In the current study, after exclusion of patients who died within 90 days following 
After propensity socre matching

a



Margin 5-9 mm

b



Margin 1-4 mm

C



Minor $\rightarrow$ Major

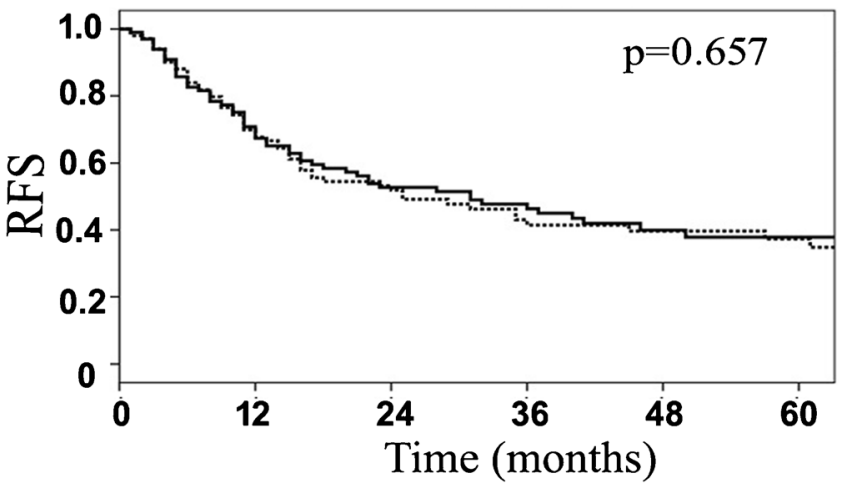

Minor

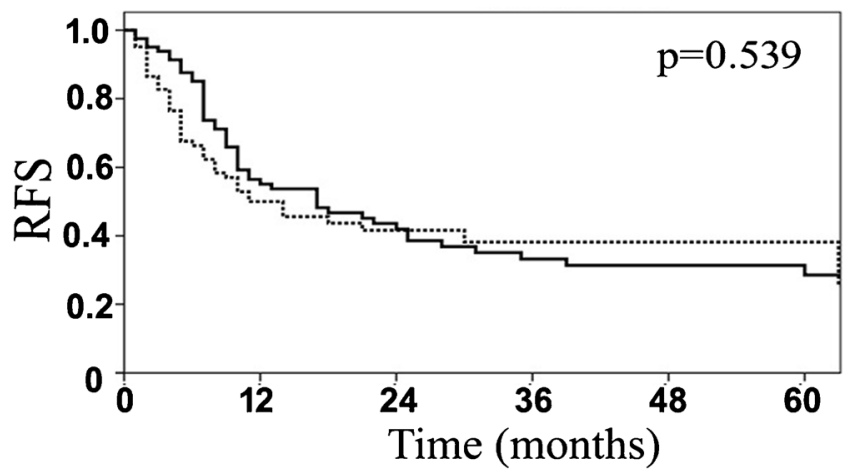

Minor a Major



Fig. 2 Overall survival $(O S)$ and recurrence-free survival $(R F S)$ of patients undergoing major and minor hepatectomy with surgical margin $\geq 10 \mathrm{~mm}(\mathbf{a})$, 5-9 $\mathrm{mm}(\mathbf{b})$, and 1-4 $\mathrm{mm}$ (c) after propensity score matching

surgery, postoperative complications were not independently associated with either OS or RFS. Examination of the Kaplan-Meier curves suggested that the impact of major complications on survival was primarily in the immediate post-operative period rather than in the long term. These data suggest that the increased complications associated with major hepatectomy may have an adverse short-term effect on mortality risk.

When matched for tumor characteristics, surgical margin width, postoperative complications, and mortality, patients 
who underwent major hepatectomy had a comparable OS and RFS compared with patients undergoing minor resection. Our previous work had demonstrated that surgical margin width was associated with long-term outcomes. ${ }^{8}$ Interestingly, in the current study, patients undergoing major hepatectomy were more likely to have an R1 margin. This finding might be explained by the fact that patients with larger and more difficult tumors more frequently underwent a major resection. However, among patients who had an R0 resection, surgical margin width was not different for patients undergoing a major versus minor resection. On subgroups analysis stratifying patients according to margin width, patients undergoing major resection had similar outcomes to patients undergoing minor resection, as long as the surgical margin was wider than $5 \mathrm{~mm}$. In contrast, when the surgical margin was narrow (1-4 mm), major resection was associated with a decrease in tumor recurrence compared with minor resection. One possible explanation is that ICC arises from intrahepatic biliary ducts and tends to disseminate into the portal venous system. Major hepatectomy may be more likely to remove the entire unilobar portal venous drainage of the involved lobe of the liver. However, unlike HCC, ICC mostly originates in a noncirrhotic liver and is therefore less likely to be multi-centric. As such, the potential superiority of major hepatectomy in eradicating microscopic metastatic lesions might be less when the surgical margin is adequate $(\geq 5 \mathrm{~mm})$. However, when the closest margin of tumor is narrow $(<5 \mathrm{~mm})$, major hepatectomy may have an effect of eradicating microscopic metastatic lesions as evidenced by decreased tumor recurrence.

There were several limitations of the current study. First, as a retrospective study, there was selection bias regarding patients undergoing major or minor resection. To mitigate this bias, we utilized PSM to create groups that were wellmatch on known prognostic risk factors (e.g., tumor size, number, vascular invasion, surgical margin, and postoperative morbidity). We were also unable to discern "true" anatomic versus non-anatomic resections at the sub-lobar level, as strict anatomic segmentectomy is not commonly performed in most institutions. Therefore, it was not possible to define and compare all "true" anatomic and nonanatomic resections.

In conclusion, extent of liver resection was not a driving factor affecting long-term outcome of patients with ICC. However, tumor factors and surgical margin impacted long-term outcomes. Importantly, major hepatectomy was associated with increased perioperative mortality and morbidity. Taken together, the data suggest that major hepatectomy for ICC does not provide a survival benefit, yet is associated with increased perioperative morbidity. Margin width, rather than the extent of resection, did impact longterm outcomes, and radical parenchymal-sparing resection should be advocated if a margin clearance of $\geq 5 \mathrm{~mm}$ can be achieved.
Acknowledgements X.-F. Z. was supported in part by the China Scholarship Council.

\section{References}

1. Nathan H, Pawlik TM. Staging of intrahepatic cholangiocarcinoma. Curr Opin Gastroenterol 2010; 26(3):269-73.

2. Mavros MN, Economopoulos KP, Alexiou VG, et al. Treatment and prognosis for patients with intrahepatic cholangiocarcinoma: systematic review and meta-analysis. JAMA Surg 2014; 149(6): 565-74.

3. Ronnekleiv-Kelly SM, Pawlik TM. Staging of intrahepatic cholangiocarcinoma. Hepatobiliary Surg Nutr 2017; 6(1):35-43.

4. Saha SK, Zhu AX, Fuchs CS, et al. Forty-year trends in cholangiocarcinoma incidence in the U.S.: intrahepatic disease on the rise. Oncologist 2016; 21(5):594-9.

5. Bridgewater J, Galle PR, Khan SA, et al. Guidelines for the diagnosis and management of intrahepatic cholangiocarcinoma. $J$ Hepatol 2014; 60(6):1268-89.

6. Zhou XD, Tang ZY, Fan J, et al. Intrahepatic cholangiocarcinoma: report of 272 patients compared with 5,829 patients with hepatocellular carcinoma. J Cancer Res Clin Oncol 2009; 135(8):107380 .

7. Brown KM, Parmar AD, Geller DA. Intrahepatic cholangiocarcinoma. Surg Oncol Clin N Am 2014; 23(2):231-46.

8. Spolverato G, Yakoob MY, Kim Y, et al. The impact of surgical margin status on long-term outcome after resection for intrahepatic cholangiocarcinoma. Ann Surg Oncol 2015; 22(12):4020-8.

9. Spolverato G, Kim Y, Alexandrescu S, et al. Management and outcomes of patients with recurrent intrahepatic cholangiocarcinoma following previous curative-intent surgical resection. Ann Surg Oncol 2016; 23(1):235-43.

10. Spolverato G, Ejaz A, Kim Y, et al. Tumor size predicts vascular invasion and histologic grade among patients undergoing resection of intrahepatic cholangiocarcinoma. J Gastrointest Surg 2014; 18(7):1284-91.

11. Hyder O, Hatzaras I, Sotiropoulos GC, et al. Recurrence after operative management of intrahepatic cholangiocarcinoma. Surgery 2013; 153(6):811-8.

12. Terminology Committee of the IHPBA. Terminology of liver anatomy and resections. HPB (Oxford) 2000; 2:333-339.

13. Ubink I, Jongen JM, Nijkamp MW, et al. Surgical and oncologic outcomes after major liver surgery and extended hemihepatectomy for colorectal liver metastases. Clin Colorectal Cancer 2016; 15(4): e193-e198.

14. Yasui M, Harada A, Torii A, et al. Impaired liver function and longterm prognosis after hepatectomy for hepatocellular carcinoma. World J Surg 1995; 19(3):439-43.

15. Nadig DE, Wade TP, Fairchild RB, et al. Major hepatic resection. Indications and results in a national hospital system from 1988 to 1992. Arch Surg 1997; 132(2):115-9.

16. Dahiya D, Wu TJ, Lee CF, et al. Minor versus major hepatic resection for small hepatocellular carcinoma (HCC) in cirrhotic patients: a 20-year experience. Surgery 2010; 147(5):676-85.

17. Regimbeau JM, Kianmanesh R, Farges O, et al. Extent of liver resection influences the outcome in patients with cirrhosis and small hepatocellular carcinoma. Surgery 2002; 131(3):311-7.

18. Stewart GD, O'Suilleabhain CB, Madhavan KK, et al. The extent of resection influences outcome following hepatectomy for colorectal liver metastases. Eur J Surg Oncol 2004; 30(4):370-6.

19. Couinaud C. Liver anatomy: portal (and suprahepatic) or biliary segmentation. Dig Surg 1999; 16(6):459-67. 
20. Edge S BD, Compton CC, Fritz AG, Greene FL, Trotti A. AJCC cancer staging manual. 7th ed. New York: Springer, 2010.

21. Dindo D, Demartines N, Clavien PA. Classification of surgical complications: a new proposal with evaluation in a cohort of 6336 patients and results of a survey. Ann Surg 2004; 240(2): 205-13.

22. de Jong MC, Nathan H, Sotiropoulos GC, et al. Intrahepatic cholangiocarcinoma: an international multi-institutional analysis of prognostic factors and lymph node assessment. J Clin Oncol 2011; 29(23):3140-5.

23. Bagante F, Spolverato G, Cucchetti A, et al. Defining when to offer operative treatment for intrahepatic cholangiocarcinoma: a regretbased decision curves analysis. Surgery 2016; 160(1):106-17.

24. Fan ST, Lo CM, Liu CL, et al. Hepatectomy for hepatocellular carcinoma: toward zero hospital deaths. Ann Surg 1999; 229(3): $322-30$.

25. Torzilli G, Makuuchi M, Inoue K, et al. No-mortality liver resection for hepatocellular carcinoma in cirrhotic and noncirrhotic patients: is there a way? A prospective analysis of our approach. Arch Surg 1999; 134(9):984-92.

26. Lang BH, Poon RT, Fan ST, et al. Perioperative and long-term outcome of major hepatic resection for small solitary hepatocellular carcinoma in patients with cirrhosis. Arch Surg 2003; 138(11): 1207-13.

27. Poon RT, Fan ST, Lo CM, et al. Long-term prognosis after resection of hepatocellular carcinoma associated with hepatitis B-related cirrhosis. J Clin Oncol 2000; 18(5):1094-101.
28. Simonetti RG, Camma C, Fiorello F, et al. Hepatocellular carcinoma. A worldwide problem and the major risk factors. Dig Dis $\mathrm{Sci}$ 1991; 36(7):962-72.

29. Farges O, Malassagne B, Flejou JF, et al. Risk of major liver resection in patients with underlying chronic liver disease: a reappraisal. Ann Surg 1999; 229(2):210-5.

30. Kumada T, Nakano S, Takeda I, et al. Patterns of recurrence after initial treatment in patients with small hepatocellular carcinoma. Hepatology 1997; 25(1):87-92.

31. Kaibori M, Matsui Y, Hijikawa T, et al. Comparison of limited and anatomic hepatic resection for hepatocellular carcinoma with hepatitis C. Surgery 2006; 139(3):385-94.

32. Karanjia ND, Lordan JT, Quiney N, et al. A comparison of right and extended right hepatectomy with all other hepatic resections for colorectal liver metastases: a ten-year study. Eur J Surg Oncol 2009; 35(1):65-70.

33. Moris D, Ronnekleiv-Kelly S, Rahnemai-Azar AA, Felekouras E, Dillhoff M, Schmidt C, Pawlik TM. Parenchymal-Sparing Versus Anatomic Liver Resection for Colorectal Liver Metastases: a Systematic Review. J Gastrointest Surg 2017; 21(6):1076-1085. doi:10.1007/s11605-017-3397-y.

34. Grazi GL, Ercolani G, Pierangeli F, et al. Improved results of liver resection for hepatocellular carcinoma on cirrhosis give the procedure added value. Ann Surg 2001; 234(1):71-8.

35. Spolverato G, Yakoob MY, Kim Y, et al. Impact of complications on long-term survival after resection of intrahepatic cholangiocarcinoma. Cancer 2015; 121(16):2730-9. 